\title{
Scale Invariance in a Perturbed Einstein-de Sitter Cosmology
}

\author{
Elcio Abdalla*, Roya Mohayaee ${ }^{\dagger}$, and Marcelo B. Ribeiro ${ }^{\ddagger}$
}

* Instituto de Física, Universidade de São Paulo - USP, CxP 66318, CEP 05315-970, São Paulo, Brazil; E-mail: eabdalla@fma.if.usp.br

$\dagger$ Dipartimento di Fisica, Universitá degli studi di Roma "La Sapienza" 5, Piazzale Aldo Moro - 100185, Roma, Italy; E-mail: roya@titanus.roma1.infn.it

$\ddagger$ Instituto de Física, Universidade do Brasil - UFRJ, CxP 68532, CEP 21945-970, Rio de Janeiro, Brazil; E-mail: mbr@if.ufrj.br

\begin{abstract}
This paper seeks to check the validity of the apparent fractal conjecture (Ribeiro 2001ab), which states that the observed power-law behaviour for the average density of large-scale distribution of galaxies arises when some observational quantities, selected by their relevance in average density profile determination, are calculated along the past light cone. Since general relativity states that astronomical observations are carried out in this spacetime hypersurface, observables necessary for direct comparison with astronomical data must be calculated along it. Implementing this condition in the proposed set of observational relations profoundly changes the behaviour of many observables in the standard cosmological models. In particular, the average density becomes inhomogeneous, even in the spatially homogeneous spacetime of standard cosmology, change which was already analysed by Ribeiro (1992b, 1993, 1994, 1995) for a non-perturbed model. Here we derive observational relations in a perturbed Einstein-de Sitter cosmology by means of the perturbation scheme proposed by Abdalla and Mohayaee (1999), where the scale factor is expanded in power series to yield perturbative terms. The differential equations derived in this perturbative context, and other observables necessary in our analysis, are solved numerically. The results show that our perturbed Einstein-de Sitter cosmology can be approximately described by a decaying power-law like average density profile, meaning that the dust distribution of this cosmology has a scaling behaviour compatible with the power-law profile of the density-distance correlation observed in the galaxy catalogues. These results show that, in the context of this work, the apparent fractal conjecture is correct.
\end{abstract}

\section{Introduction}

It has been known since 1970 that the large-scale average density mass distribution of the Universe, constructed with astronomical data on the galaxy distribution, decreases linearly 
with increasing distances, following a power-law pattern in a log-log plot, and with an exponent in the range from 1.7 to 2 (Kihara and Saki 1970; de Vaucouleurs 1970, and references therein). Further observational and theoretical analysis of this behaviour, made in the 1970's by Peebles and collaborators (see Peebles 1980, and references therein), interpreted this effect as being a result of small scale density perturbations necessary for the formation of structures in the Universe. Since then it has been a more or less conventional wisdom to suppose that such a power-law density profile arises as a result of these density fluctuations, an effect being short range in nature, and doomed to disappear at higher distances where the homogeneity predicted by the Friedmann cosmological models is supposed to be observed.

This conventional picture was, however, challenged in 1987 by L. Pietronero, who, taking earlier suggestion advanced by Mandelbrot (see Mandelbrot 1983, and references therein), and which was also discussed by Peebles (1980), proposed that the luminous large-scale matter distribution in the Universe should follow a scale invariant pattern, arising from the underlying smoothed-out and averaged fractal galaxy distribution system.

Pietronero's (1987) paper had the effect of starting a sharp controversy in the literature between the proponents and opposers of this "fractal universe" (see Turok 1997 and references therein). This controversy is so far mainly focused on the issues of whether or not observations of large-scale galaxy distribution support or dismiss an average density powerlaw profile that decays at increasing distance, and the depth of this scale invariant system (see the reviews by Ribeiro and Miguelote 1998, Sylos-Labini et al. 1998, and references therein). Although, a consensus on these points is yet to be achieved, it is clear by now that settling, or even clarifying, those controversial points has become an important issue in cosmology, inasmuch as this "fractal debate" has already reached the main stream of cosmological research (Turok 1997; Coles 1998; Wu, Lahav and Rees 1999; Martínez 1999).円 In any case, at very short redshifts (for interstellar medium, and clusters of galaxies) we know that there is a scale invariant structure.

Since this is a debate in cosmology, it is only natural that the feasibility of some kind of scale invariant universe model should also be investigated in a relativistic framework, and, therefore, relativistic aspects of cosmological models bearing average density scaling features

1 Pietronero's (1987) article is only the most recent form in which the old idea that matter in the Universe is structured in a hierarchical manner has resurfaced. For instance, following de Vaucouleurs' (1970) case for a hierarchical universe, Wertz (1970, 1971) advanced a model mathematically identical to Pietronero's (1987), where a discussion about scaling in galaxy clustering can already be found. However, as scale invariant ideas had not yet appeared, Wertz was unable to reach many important conclusions found later, and independently, by Pietronero, in special the significance of the fractal dimension in galaxy clustering, and the proposal of statistical tools able to appropriately describe a smoothed-out hierarchical pattern. That may explain why Wertz's work has remained largely ignored so far. Unaware of Wertz's work, but inspired by de Vaucouleurs, Mandelbrot (1983) revived the hierarchical universe model and made a thorough discussion about the scaling properties of galaxy distribution, fully characterizing it as a scale invariant structure. A discussion about the similarities and differences between Wertz's and Pietronero's approaches to the problem of universal hierarchical clumping of matter can be found in Ribeiro and Miguelote (1998; see also Ribeiro 1994). 
can be expected to play an important role in this debate.

Some relativistic cosmologies of this type have already been proposed in the LemaitreTolman-Bondi (LTB) spacetime, which is the most general spherically symmetric dust solution to Einstein's field equations (Ribeiro 1992a, 1993, 1994; Matravers 1998; Humphreys, Matravers and Marteens 1998), but a potentially important investigation is the possibility of appearance of scale invariant features in Friedmann cosmologies with small scales perturbations. If average densities with decaying power-law like profiles could somehow appear in the standard cosmological models, many points of the current debate about the density behaviour of galaxy distribution could be clarified, or even resolved, if a relativistic perspective is taken for these effects.

In a previous paper, one of us (Ribeiro 1992b) studied observational relations in a nonperturbed Einstein-de Sitter (EdS) cosmology, that is, without any type of metric or density perturbation, and the general conclusions were that this cosmology does not show scaling features along the past light cone, in the sense of not having a power law decrease of the average density at increasing distances, as predicted in Pietronero's (1987) original model. However, since all calculations took the backward null geodesic into consideration it was clearly demonstrated that this model does not show up as observationally homogeneous either, even at small redshifts $(z \approx 0.04)$, and this result is a consequence of the fact that the homogeneity of the standard cosmological models is spatial, that is, it is a geometrical feature which does not necessarily translate itself into an astronomically observable quantity. Although a number of authors are aware of this fact, what came as a surprise had been the low value for the redshift where this observational inhomogeneity appears. Therefore, it was clear by then that relativistic effects start to play an important role in observational cosmology at much lower redshift values than previously assumed. Nonetheless, at least part of these scaling features can be analysed in terms of a purely non-relativistic model, as done in Abdalla et al. (1999), though in such a case direct comparison with observational data is more difficult.

In a sequel paper (Ribeiro 1995), this result was further analysed and the reasons why this relativistic effect seems to have been overlooked in the literature was clarified. Due to the non-linearity of the Einstein field equations, observational relations behave differently at different redshift depths. Thus, while the linearity of the Hubble law is well preserved in the EdS model up to $z \approx 1$, a value implicitly assumed by many other authors as the lower limit up to where relativistic effects could be safely ignored, the density is strongly affected by relativistic effects at much lower redshift values. A power series expansion of these two quantities showed that while the zeroth order term vanishes in the distance-redshift relation, it is non-zero for the average density as plotted against redshift. This zeroth order term is the main reason for the different behaviour of these two observational quantities at small redshifts. Pietronero et al. (1997) referred to this effect as the "Hubble-de Vaucouleurs paradox", however, from the discussion above it is clear that there is no paradox. Indeed what seems to be a paradox are just very different relativistic effects on the observables at 
the moderate redshift range $(0.1 \leq z<1)$.

Similar effects of departures from the expected Euclidean results at small redshifts were also reported by Longair (1995, p. 398), and the starting point for his findings was the same as that of Ribeiro (1992b, 1995): the use of source number count expression along the null cone. Nevertheless, the path followed by Longair was quite different from Ribeiro's. While the former kept his conclusions essentially qualitative and did not make further investigations of the consequences of this effect in other observational quantities, like the two-point correlation function, or provided an explanation for the underlying reasons for this effect, the latter attempted to address all these issues (see details in Ribeiro 1995).

Despite these interesting and encouraging results, they must still be considered as preliminary, inasmuch as the analysis advanced by Ribeiro (1992b, 1995) was carried out in an unperturbed model, and, therefore, some of its features are unrealistic, especially the behaviour of the observational quantities at very small $z$, where the average density tends to a constant value. In addition, one important question remained. While Ribeiro (1992b) showed that an unperturbed EdS model does not have scale invariant features, in the sense of not having a power law decay of the average density at increasing depths, it, nevertheless, also showed very clearly that there is indeed a strong decay of the average density at increasing values of the luminosity distance or the redshift, an effect termed by Ribeiro (1995) as "observational inhomogeneity of the standard model". 2f Bearing this result in mind, it is only natural to ask whether or not a perturbed model could turn the density decay at increasing redshift depths into a power law type decay, as predicted, and claimed to be observed, by the scale invariant description of galaxy clustering (Pietronero 1987; Coleman and Pietronero 1992; Pietronero et al. 1997; Ribeiro and Miguelote 1998; Sylos-Labini et al. 1998; Pietronero and Sylos-Labini 2000).

It must be clearly understood that the effects described above appear not simply by carrying out calculations along the null cone, but by doing this by means of a set of observational relations whose original purpose was power-law density profile characterization, and which turned out to be more useful than originally envisaged. That was fully explained in Ribeiro $(1993,1994,1995)$, and it implies that if one is simply doing calculations along the null cone it is most probable that one will find no scaling pattern of any kind at all. 5 Thus, to even start considering scale invariance in relativistic cosmology it was necessary to adapt the original analytical tools proposed by Pietronero (1987) into a relativistic framework, and when doing this it became clear that the chosen set of observational quantities had to have their behaviour studied along the past null cone (Ribeiro 1992a). Therefore, the surprising

2 Note that this result is achieved only when an appropriately chosen set of observational quantities is calculated by taking fully into account that light rays follow null geodesics, as stated by general relativity. Some authors do not get this same result because they do not take this relativistic fact into consideration, and/or use different, or inappropriate, observational quantities (see details in Ribeiro 2001b).

3 See also Ribeiro (2001b) for the pitfalls of such a simplistic approach when dealing with the problem of the possible observational smoothness of the Universe. 
results stated in the previous paragraphs could only have appeared through a mix of the use of a specific set of relativistically adapted observational relations, and the realization that even at small scales $(z<0.1)$ relativistic effects start to play an important role in cosmology.

The apparent fractal conjecture, as advanced by Ribeiro (2001ab), states essentially that the observed power-law average density profile of large-scale galaxy distribution should be a consequence of the fact that astronomical observations are made on the backward null cone, and, therefore, observational quantities necessary for scale invariant characterization must be calculated accordingly. If we take together these two considerations into account in small scales perturbed standard models, then we should find a scaling pattern on average density in the sense of power-law density profiles. If this hypothesis proves, even partially, correct, many of the discrepancies between both sides of the above mentioned debate could be immediately resolved, and without the need of bringing into question the standard cosmological model or even the cosmological principle. Moreover, it is important to mention that galaxy catalogues have for some time been showing a power-law behaviour for the density-distance correlation (Davis et al. 1988; Geller 1989), and, therefore, this average density power-law profile for the galaxy distribution could be explained by this conjecture.

This paper seeks to prove whether or not Ribeiro's (2001ab) conjecture is correct, at least in a narrow sense. Our aim here is to investigate if a perturbed standard cosmological model could show scaling features similar to the power-law density profile predicted by Pietronero and collaborators.

Here we show that by starting from the simplest possible cosmological model, EdS, and carrying out a specific metric perturbation appropriate to our needs, the conjecture is correct under an approximation which is very reasonable if we consider the large error margins produced by astronomical observations. We followed the perturbative scheme proposed by Abdalla and Mohayaee (1999), where the scale factor is expanded in power series to yield perturbative terms. However, in order to use this scheme we had first to derive observational relations along the past null cone and then relate the results with actual observations as obtained in astronomy. Then we found numerical solutions which show fractal like scaling features, in the sense of Pietronero (1987).

The paper is organized as follows. In section 2 we summarize the perturbative method used here and present the perturbed EdS spacetime. Section 3 deals with calculating the various observational relations in the chosen spacetime, and section 4 discusses the numerical scheme which is used to obtain numerical solutions for the observational quantities. Section 5 shows the numerical results obtained, and how a scale invariant pattern appears from these results. The paper finishes with a conclusion. 


\section{The Perturbed Metric}

Let us start with the inhomogeneous spherically symmetric metric as proposed by Abdalla and Mohayaee (1999),

$$
d S^{2}=-d t^{2}+R^{2}(r, t)\left[\frac{d r^{2}}{f^{2}(r)}+r^{2} d \Omega^{2}\right]
$$

where

$$
d \Omega^{2}=d \theta^{2}+\sin ^{2} \theta d \phi^{2}
$$

and

$$
f^{2}(r)=1-k r^{2} ; \quad k=0, \pm 1 .
$$

The proposal is to solve Einstein's field equations,

$$
R_{a b}-\frac{1}{2} g_{a b} R=-8 \pi G T_{a b}
$$

for a perfect fluid universe with the metric above, but by means of series expansions of the form,

$$
R(r, t)=\sum_{n=0}^{\infty} \frac{R_{n}(t)}{r^{n}}, \quad \rho(r, t)=\sum_{n=0}^{\infty} \frac{\rho_{n}(t)}{r^{n}}, \quad p(r, t)=\sum_{n=0}^{\infty} \frac{p_{n}(t)}{r^{n}} .
$$

The zeroth order terms in this expansion are of unperturbed standard cosmologies. Thus, $R_{0}(t)$ is the scale factor of the Friedmann universe.

As a first approach to modelling a smoothed-out and averaged fractal system in the standard cosmology, both the metric (1) and its perturbation scheme, given by equations (5), are well suited for the purposes of this work, inasmuch as all previous relativistic fractal cosmologies have so far been proposed in the LTB spacetime (see $\S 1$ above). This means that equations (11) and (5) are special cases of the LTB metric (Ribeiro 1992a), making it possible to compare the results of this paper with the relativistic fractal cosmologies already known. In addition, it must be noted that here we are taking an operative definition of fractality, which refers to the property shown by the observed large-scale distribution of galaxies of having an average density power-law type decay at increasing distances. So, in this paper fractality means in fact observational fractality, in the astronomical sense, and only resembles non-analytical fractal sets in the sense that if we define a smooth-out average density on those sets, the properties of this average density are similar to what is found in observational cosmology data. In other words, they are both of power-law type ones. Therefore, under this operative definition, we can talk about fractality, or fractal properties, in completely smooth relativistic cosmological models, where the cosmological fluid approximation is assumed (Ribeiro 2001b).

For flat matter dominated universe $(p=0, k=0)$, that is, for perturbed EdS cosmology, the metric

$$
d S^{2}=-d t^{2}+R^{2}(r, t)\left(d r^{2}+r^{2} d \Omega^{2}\right)
$$


produces solutions of the field equations with perturbative terms that represent growth of inhomogeneities. These, to first order, are

$$
\begin{gathered}
R(r, t)=A t^{2 / 3}+\left(\frac{-9 C_{1}}{10 A^{2}}\right) \frac{t^{4 / 3}}{r^{3}}, \\
\rho(r, t)=\frac{1}{6 \pi G t^{2}}+\left(\frac{-3 C_{1}}{10 \pi G A^{3}}\right) \frac{t^{-4 / 3}}{r^{3}},
\end{gathered}
$$

where $A$ and $C_{1}$ are constants. The first terms of the right hand side of equations (7) and (8) are of unperturbed Friedmann universe and the two additional terms in both equations represent the first perturbative inhomogeneous corrections which yield growing modes. Terms corresponding to decaying modes are ignored here. This perturbative solution reproduces standard results, such as found in Weinberg (1972), and it corresponds to a soft perturbation, which is almost homogeneous at large values of the coordinate $r$ (see details in Abdalla and Mohayaee 1999). There are additional perturbative terms which also yield growing modes, but in order to try a first check of the possible validity of the apparent fractal conjecture, we only need the simplest perturbative model. Therefore, at this stage we will ignore other terms in the series.

We shall need for later usage the time derivative of equation (7),

$$
\frac{\partial R}{\partial t}=\frac{2}{3} A t^{-1 / 3}+\left(\frac{-6 C_{1}}{5 A^{2}}\right) \frac{t^{1 / 3}}{r^{3}} .
$$

As a final remark, while the proposed perturbation is the most convenient for the purposes of this work, as explained above, it remains to be seen whether or not other types of perturbations could also be well, or better, suited for checking the validity of the apparent fractal conjecture. We shall not pursue this investigation here.

\section{Observational Relations Along the Past Null Cone}

The first step towards obtaining observational relations in the spacetime given by metric (1) is taken by solving its past radial null geodesic. This astronomically important hypersurface provides the geometrical locus for light rays that travel towards us. It is obtained when we take $d S^{2}=d \theta^{2}=d \phi^{2}=0$ in metric (1). In this way we obtain the following expression,

$$
\frac{d t}{d r}=-\frac{R}{f} .
$$

Note that it is just a matter of convenience to write the past radial null geodesic above as having the radial coordinate $r$ as its parameter. In fact, both $r$ and $t$ coordinates are functions of the null cone affine parameter $\lambda$, which means that the equation above may also be alternatively written as

$$
\frac{d t}{d \lambda}=-\frac{R}{f} \frac{d r}{d \lambda}
$$

It is rather a difficult task to obtain an analytical solution for the null geodesic (10) in the flat matter dominated case $\left(f^{2}=1\right)$, or equivalently, to derive analytical expressions for 
the observational relations along the past light cone for the perturbative solutions (7) and (8). Thus, we choose an alternative approach. We first derive all the necessary observational relations for metric (1), and then solve the problem numerically to obtain solutions corresponding to the perturbation of the metric (6), as given by equations (4) and (8). Such a procedure will eventually allow us to obtain the desired observational relations, although in numerical form. To pursue this path, we should start by deriving the redshift in the geometry given by equation (1).

The general expression for the redshift, in any spacetime, is given by (see, e.g., Ellis 1971)

$$
1+z=\frac{\left(u^{a} k_{a}\right)_{\text {source }}}{\left(u^{a} k_{a}\right)_{\text {observer }}}
$$

where $u^{a}$ is the 4 -velocity of source and observer and $k^{a}$ is the tangent vector of the null geodesic joining them. If source and observer are comoving, then $u^{a}=\delta_{0}^{a}$, and equation (12) becomes

$$
1+z=\frac{(d t / d \lambda)_{\text {source }}}{(d t / d \lambda)_{\text {observer }}}
$$

since, $g_{00}=-1$, and, by definition, $k^{0}=d t / d \lambda$.

Finding $(d t / d \lambda)$ at both source and observer requires the use of an indirect method, which will be described as follows. We start with the Lagrangian for the radial metric,

$$
\mathcal{L}=-\left(\frac{d t}{d \lambda}\right)^{2}+\frac{R^{2}(r, t)}{f^{2}(r)}\left(\frac{d r}{d \lambda}\right)^{2}
$$

The Euler-Lagrange equations of motion

$$
\frac{d}{d \lambda} \frac{\partial \mathcal{L}}{\partial \dot{q}}-\frac{\partial \mathcal{L}}{\partial q}=0
$$

can be applied to equation (14), yielding

$$
\begin{gathered}
\frac{d \dot{t}}{d \lambda}+\left(\frac{R}{f^{2}} \frac{\partial R}{\partial t}\right) \dot{r}^{2}=0 \\
\frac{d \dot{r}}{d \lambda}+\frac{1}{R}\left(\frac{\partial R}{\partial r}-\frac{R}{f} \frac{d f}{d r}\right) \dot{r}^{2}+\left(\frac{2}{R} \frac{\partial R}{\partial t}\right) \dot{r} \dot{t}=0,
\end{gathered}
$$

where the dot means derivative with respect to the affine parameter $\lambda$. If we use the null geodesic (11) in equations (16) and (17), they can both be integrated once. The results may be respectively written as

$$
\begin{gathered}
\dot{t}=\left[\int\left(\frac{1}{R} \frac{\partial R}{\partial t}\right) d \lambda+b_{1}\right]^{-1}, \\
\dot{r}=\left[\int\left(\frac{1}{R} \frac{\partial R}{\partial r}-\frac{1}{f} \frac{d f}{d r}-\frac{2}{f} \frac{\partial R}{\partial t}\right) d \lambda+b_{2}\right]^{-1},
\end{gathered}
$$

where $b_{1}$ and $b_{2}$ are integrations constants.

To find those constants, let us now write a 2 -surface displacement, with $t$ and $\phi$ constants, of metric (11),

$$
d S^{2}=\frac{R^{2}}{f^{2}}\left(d r^{2}+f^{2} r^{2} d \theta^{2}\right)
$$


We shall now require the metric to be regular at the spatial origin, that is, as $r \rightarrow 0$ the metric must be Euclidean. Therefore, $f^{2} \rightarrow 1, R^{2} / f^{2} \rightarrow$ constant, as $r \rightarrow 0$. In other words, we are requiring that metric (1) should obey the central regularity condition (Bonnor 1974; Ribeiro 1993; Humphreys, Matravers and Marteens 1998),

$$
\lim _{r \rightarrow 0} R=1 .
$$

Now, if we re-substitute solutions (18) and (19) back into the null geodesic (11) we obtain,

$$
\left[\int\left(\frac{1}{R} \frac{\partial R}{\partial r}-\frac{1}{f} \frac{d f}{d r}-\frac{2}{f} \frac{\partial R}{\partial t}\right) d \lambda+b_{2}\right]=-\frac{R}{f}\left[\int\left(\frac{1}{R} \frac{\partial R}{\partial t}\right) d \lambda+b_{1}\right] .
$$

This equation is valid for any $\lambda$, including at the origin, where the observer is located. From now on we will be labelling the event of observation as $r=\lambda=0$. So, considering the regularity condition (21), equation (22) may be written as,

$$
b_{2}=-b_{1}
$$

If we now consider the same regularity conditions, then equation (18) becomes,

$$
\left[\frac{d t}{d \lambda}\right]_{\lambda=0}=\frac{1}{b_{1}}
$$

Inasmuch as, we are interested in incoming light rays, i.e., our model deals with photons along the past light cone, it is natural to choose $b_{1}=-1$ as the value for this constant. Therefore,

$$
b_{1}=-1, \quad \Rightarrow \quad b_{2}=1,
$$

and we may write equations (18) and (24) as,

$$
\begin{gathered}
\frac{d t}{d \lambda}=\left[\int\left(\frac{1}{R} \frac{\partial R}{\partial t}\right) d \lambda-1\right]^{-1}, \\
{\left[\frac{d t}{d \lambda}\right]_{\lambda=0}=-1 .}
\end{gathered}
$$

If we now define an auxiliary term, named as I-term, as being given by,

$$
I \equiv \int\left(\frac{1}{R} \frac{\partial R}{\partial t}\right) d \lambda
$$

equations (26) and (27) allow us to re-write the redshift (13) as follows,

$$
z=\frac{I}{1-I}
$$

We still have to calculate the I-term in order to evaluate the redshift, and this can be done as follows. Considering equations (25) and (28) we may re-write equation (22) as,

$$
\left\{\int\left[\frac{1}{R} \frac{\partial R}{\partial r}-\frac{1}{f} \frac{d f}{d r}-\frac{2}{f} \frac{\partial R}{\partial t}\right] d \lambda+1\right\}=-\frac{R}{f}(I-1) .
$$


Thus, considering equations (28) and (30), equations (18) and (19) may be re-written as,

$$
\begin{gathered}
\frac{d t}{d \lambda}=\frac{1}{I-1}, \\
\frac{d r}{d \lambda}=\frac{f}{(1-I) R} .
\end{gathered}
$$

From equation (28) it is easy to see that,

$$
\frac{d I}{d r}=\frac{d}{d r} \int \frac{1}{R} \frac{\partial R}{\partial t} \frac{d \lambda}{d r} d r
$$

and, by substituting equation (32) in the expression above, we finally obtain,

$$
\frac{d I}{d r}=\left(\frac{1-I}{f}\right) \frac{\partial R}{\partial t}
$$

The solution of the first order ordinary differential equation (34) allows us to calculate the I-term and, as a consequence, the redshift, as given by equation (29). When $r \rightarrow 0$, $f=1, R=1$ and $I=0,(d I / d r)=0$. Remembering the perturbed solution (7), we are facing again a differential equation whose analytical solution is difficult, if not impossible, to find.

The other observational relations relevant to the problem under consideration can be straightforwardly calculated now. The observer area distance, or simply area distance, ff as defined by Ellis (1971) for any spacetime, is given by

$$
\left(d_{A}\right)^{2}=\frac{d A_{0}}{d \Omega_{0}}
$$

where $d \Omega_{0}$ is the solid angle element for constant $r$, and $d A_{0}$ is the cross sectional area for this solid angle (see Ellis 1971). For metric (6) we have,

$$
d \Omega_{0}=d \theta \sin \theta d \phi, \quad d A_{0}=R^{2} r^{2} \sin \theta d \theta d \phi
$$

Therefore, the area distance is given by the following expression,

$$
d_{A}=r R
$$

The luminosity distance is obtained from the area distance by means of Etherington's reciprocity theorem (1933; see also Ellis 1971; Schneider, Ehlers and Falco 1992), which relates both distance definitions through the expression

$$
\left(d_{\ell}\right)^{2}=\left(d_{A}\right)^{2}(1+z)^{4} .
$$

So, for the spacetime (6) we have

$$
d_{\ell}=r R(1+z)^{2}=\frac{r R}{(1-I)^{2}} .
$$

\footnotetext{
4 This definition of distance is the same as Weinberg's (1972) angular diameter distance, and Kristian and Sachs' (1966) corrected luminosity distance. A detailed discussion about distances in cosmology as applied to the problem discussed in here can be found in Ribeiro (2001b).
} 
The general expression for number counting in any cosmological model at a point $P$ down the null cone is given by (Ellis 1971),

$$
d N=\left(d_{A}\right)^{2} d \Omega_{0}\left[n\left(-k^{a} u_{a}\right)\right]_{P} d \lambda
$$

Here $n$ is the number density of radiating sources per unit proper volume. Considering equation (11) and that $k^{0}=d t / d \lambda$ for comoving source, equation (40) becomes,

$$
d N=4 \pi n r^{2} R^{3} d r
$$

where we have performed an integration over all solid angles. If we now make the assumption that all sources are galaxies, with approximately the same average mass, then

$$
n=\frac{\rho}{M_{g}}
$$

with $M_{g} \approx 10^{11} M_{\odot}$ being the rest mass of an average galaxy, and $\rho$ comes from equation (8), we obtain another differential equation to be solved numerically,

$$
\frac{d N}{d r}=\frac{4 \pi}{M_{g}} \rho r^{2} R^{3}
$$

Finally, to discuss fractality, in the sense of a smoothed-out and averaged scale invariant system with a decaying power-law profile for the average density, we also need the observed volume, here defined as

$$
V=\frac{4}{3} \pi\left(d_{\ell}\right)^{3}
$$

and the observed average density,

$$
\langle\rho\rangle=\frac{M_{g} N}{V} .
$$

Ribeiro (1995) showed that in an unperturbed EdS model the following relations holds,

$$
\Gamma^{*}=\frac{\langle\rho\rangle}{M_{g}}=\langle n\rangle
$$

Therefore, Pietronero's (1987) conditional average density $\Gamma^{*}$ is equal to the observed average number density in an unperturbed EdS model. Since, these two quantities are fundamental in the characterization of a single fractal structure (Ribeiro and Miguelote 1998), they can also be expected to be equally fundamental in the fractal characterization of the perturbed model considered here.

\section{Numerical Problem}

We have seen in the previous section that in order to obtain observational relations for flat matter dominated universe, we need to solve three differential equations: the past radial null geodesic (10), the I-term (34) and the cumulative source number count (43). However, in obtaining the I-term and source count we need first to solve the null geodesic, as both 
depend on the scale factor $R(r, t)$. In other words, integrating the null cone produces a solution given by the function $t=t(r)$, which is necessary for integrating the I-term and source count along the null cone.

The integration procedure outlined above can be algorithmically expressed as follows. We start with the initial conditions $r_{1}, t_{1}, I_{1}, N_{1}$, use the first two to find $R_{1}, \rho_{1},[\partial R / \partial t]_{1}$, by means of expressions (7) and (8), use some numerical code for solving ordinary differential equations to advance one step and finally find $t_{2}$, then $I_{2}, N_{2}$. As $r_{2}$ is known in advance, since it is the independent variable, the newly found values $t_{2}, I_{2}, N_{2}$ are used to repeat the cycle until we finish the integration, in $r_{n}$. Although that amounts to a simple numerical procedure, some care is needed in order to make sure we will be using the values obtained in the integration of the null geodesic to feed the evaluation of functions (7) and (8). In other words, if $t_{i}$ is an intermediary value $(i=1, \ldots, n)$, obtained numerically, of the null geodesic $t=t(r)$, then $t_{i}$ must be used to find $R_{i}, \rho_{i},[\partial R / \partial t]_{i}$, which are then used to find $t_{i+1}, I_{i+1}$, and $N_{i+1}$, and so on.

The initial values pose a problem: if we start the integration at $r=0$, according to equations (5) we will face a singularity at the origin. To avoid this difficulty we will assume a flat and Euclidean spacetime from $r=0$ up to $r=\varepsilon$, where $\varepsilon$ will be as small as necessary. As seen above, previous studies have shown that observational departures from spatial homogeneity occur at $z \approx 0.04$, which means $\sim 160$ Mpc. Therefore, it is reasonable to assume a Euclidean spacetime up to $\sim 100 \mathrm{Mpc}$, or $z \approx 0.03$, which means taking $\varepsilon=0.1$ as the initial integration value. Here we will be taking distances in Gpc and units such that $c=G=1$. In these units $M_{g}=10^{11} M_{\odot}=4.787591 \times 10^{-12}$ and $H_{0}=0.250173$ for the value of the Hubble constant of $75 \mathrm{~km} \mathrm{~s}^{-1} \mathrm{Mpc}^{-1}$ in the usual units.

The assumption above is completely coherent with the central regularity condition (21), but in fact introduces the notion that there is a Euclidean to non-Euclidean interface at $r=\varepsilon$. This means that we need to find initial values at $r=\varepsilon$ to start the integration. Then, at this interface, the null geodesic reduces to

$$
t=-r+t_{0}
$$

which implies the following initial values,

$$
\left\{\begin{array}{l}
R=1, \\
t=-\varepsilon+t_{0}, \\
r=\varepsilon,
\end{array}\right.
$$

where $t_{0}$ is the label given by the time coordinate at the present epoch. Therefore, equation (47) implies that the event of observation, that is, the "here and now", is labeled by $r=$ $\lambda=0$, and $t=t_{0}$. Since the time elapsed since the big bang singularity hypersurface is the same for all observers in the standard cosmologies, we may take $t_{0}$ to be the same as in the unperturbed EdS model, that is,

$$
t_{0}=\frac{2}{3 H_{0}} .
$$


The initial values (48), once applied to equation (7), allows us to find an expression linking the two constants $C_{1}$ and $A$,

$$
C_{1}=\frac{10}{9} A^{2} \varepsilon^{3}\left[A\left(\frac{2}{3 H_{0}}-\varepsilon\right)^{2 / 3}-1\right]\left(\frac{2}{3 H_{0}}-\varepsilon\right)^{-4 / 3} .
$$

In the flat and Euclidean region close to the origin, the universal density is assumed to be constant, whose value should be the critical density for Friedmann universe, that is, the value of the local density for a EdS universe at present time. By using this requirement in equation (8), we get,

$$
\rho_{0}=\frac{3 H_{0}^{2}}{8 \pi}=\frac{1}{6 \pi}\left(\frac{2}{3 H_{0}}-\varepsilon\right)^{-2}-\frac{3 C_{1}}{10 \pi A^{3} \varepsilon^{3}}\left(\frac{2}{3 H_{0}}-\varepsilon\right)^{-4 / 3},
$$

or, putting $C_{1}$ in evidence,

$$
C_{1}=\frac{15 A^{3} H_{0}{ }^{3} \varepsilon^{4}}{4\left(2-3 \varepsilon H_{0}\right)}\left(\frac{2}{3 H_{0}}-\varepsilon\right)^{4 / 3} .
$$

Equations (50) and (52) provide conditions for the two unknown constants $C_{1}$ and $A$ to be expressed in terms of the Hubble constant. We thus obtain,

$$
\begin{gathered}
A=\frac{8}{\left[8-3 \varepsilon H_{0}\left(2-3 \varepsilon H_{0}\right)\right]}\left(\frac{2}{3 H_{0}}-\varepsilon\right)^{-2 / 3}, \\
C_{1}=\frac{1920 H_{0}{ }^{3} \varepsilon^{4}}{\left(2-3 \varepsilon H_{0}\right)\left[8-3 \varepsilon H_{0}\left(2-3 \varepsilon H_{0}\right)\right]^{3}}\left(\frac{2}{3 H_{0}}-\varepsilon\right)^{-2 / 3} .
\end{gathered}
$$

A power series expansion for $\varepsilon$ in equation (53) yields

$$
A=\left(\frac{3 H_{0}}{2}\right)^{2 / 3}+O(\varepsilon)
$$

while in equation (54), a similar expansion produces,

$$
C_{1}=\frac{15}{8}\left(\frac{3 H_{0}}{2}\right)^{2 / 3} H_{0}{ }^{3} \varepsilon^{4}+O\left(\varepsilon^{5}\right) .
$$

Thus, if $\varepsilon$ is too small, meaning a too small flat region, then $A$ remains a nonzero constant, and $C_{1}$ becomes negligibly small. In such a case the perturbative terms in equations (7) and (8) will vanish.

Finally, for number count (43), up to $r=\varepsilon$ we have

$$
\frac{d N}{d r}=\frac{4 \pi}{M_{g}} \rho_{0} r^{2}, \quad \Longrightarrow \quad N(\varepsilon)=\frac{H_{0}^{2} \varepsilon^{3}}{2 M_{g}} .
$$

This last equation implies that,

$$
\left.\langle\rho\rangle\right|_{r=\varepsilon}=\rho_{0},
$$

as it should. 


\section{$5 \quad$ Numerical Solutions}

The previously defined procedure for computing observational relations in a perturbed model was carried out by means of a simple numerical code written in FORTRAN 77 . The results are better or worse depending on the size of the flat region, i.e., depending on the value of $\varepsilon$. For very small values, due to the fourth power for $\varepsilon$, as seen in equation (56), the perturbation tends to vanish and the results are similar to those found years ago by Ribeiro (1992b, 1993, 1994, 1995). For larger values of $\varepsilon$ the form of the curve showing the average density $\langle\rho\rangle$ as plotted against the luminosity distance $d_{\ell}$ does approach a linear behaviour in a $\log \log$ scale, meaning a power law decay for the observed average density along the past null cone. Such a behaviour means that our perturbed EdS cosmological model can be approximately described by a decaying power-law like distribution, a result which validates, in an approximate manner, the apparent fractal conjecture, at least by means of the perturbation scheme used here.

Figure 11 shows the best numerical result obtained, and there one can see that the property of a smoothed-out and averaged fractal system of exhibiting a linear decay of its average density, due to its power law feature, appears approximately. If we consider that astronomical data usually have large error margins, and that spatially flat LTB models are known at providing not so good fractal modelling (Ribeiro 1993), the results shown in figure 11 can be considered as quite good. Therefore, in spite of the simplicity of the model we have succeeded in finding an observational scaling behaviour in the EdS cosmology, and with a fractal dimension not too different from the expected value foreseen by observations (see, e.g., Ribeiro and Miguelote 1998, Sylos-Labini et al. 1998).

Previous studies with this kind of fractal modelling in LTB spacetimes showed that spatially flat models do not provide very good results, while open models provide the best ones (Ribeiro 1993, 1994; see also Humphreys, Matravers and Marteens 1998). Therefore, in view of this it is reasonable to conclude that the best results regarding the validity of the apparent fractal conjecture should come from perturbed open Friedmann cosmologies.

The situation can certainly be improved by either modifying the type of perturbation, and also (possibly more important) if we better accommodate the observed nearby matter distribution, seen here as just flat geometry.

\section{Conclusion}

In this paper, we have used a perturbative model of the Einstein-de Sitter cosmology to test the validity of the hypothesis known as "the apparent fractal conjecture" (Ribeiro 2001ab), which states that the observed power-law average density profile decay, derived from observations of large-scale distribution of galaxies, appears when observational quantities relevant for scale invariant characterization are calculated along the past light cone in universe models with small scales perturbations. These are the quantities which should be directly compared 


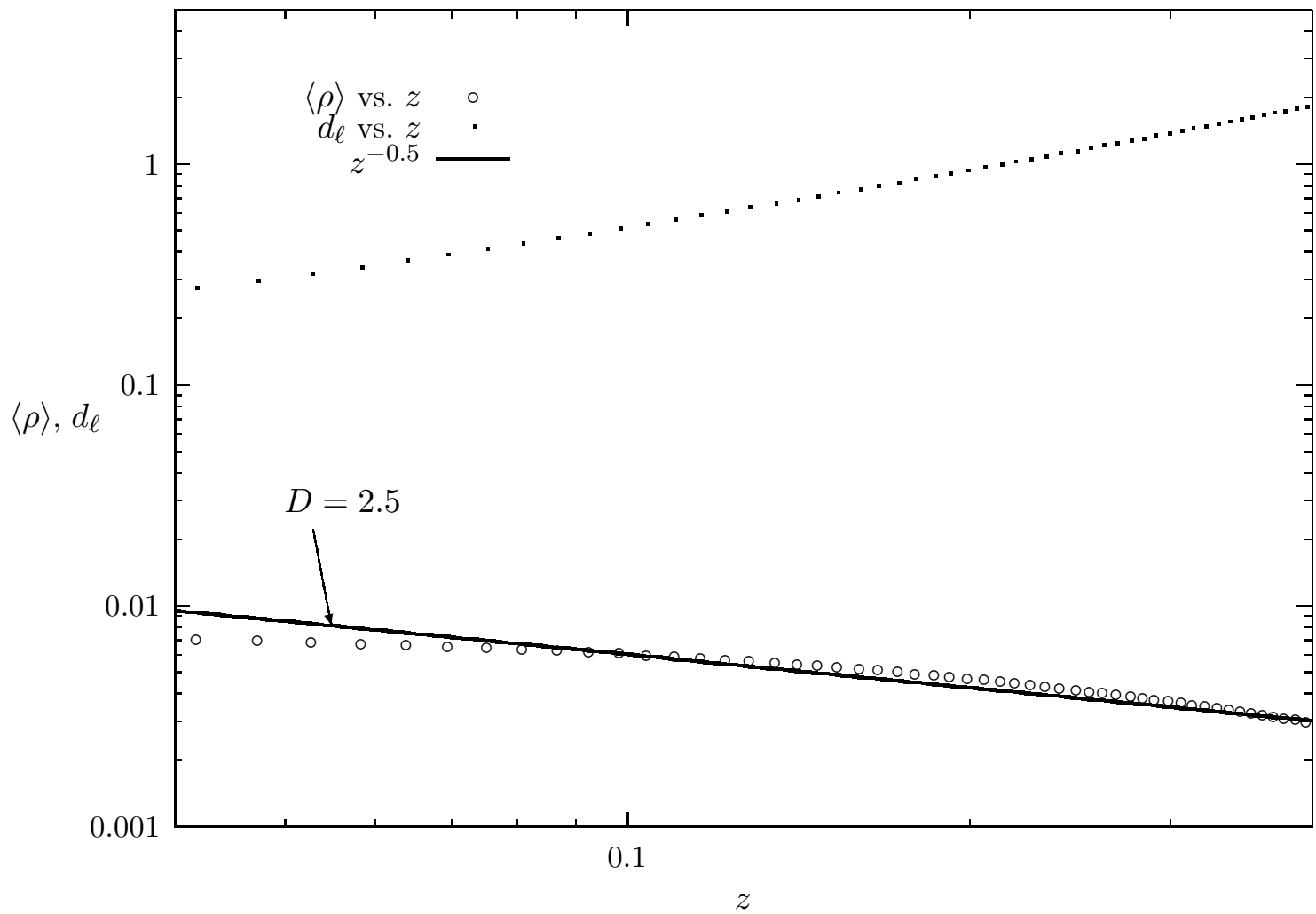

Figure 1: This figure shows a plot in the redshift range $0.04 \leq z \leq 0.4$, containing numerical results of the observational quantities of interest, namely, the observed average density $\langle\rho\rangle$, the luminosity distance $d_{\ell}$, and the redshift $z$. These observables were calculated along the past null cone, and by using numerical solutions of the differential equations (10), (34), and (43). The value used to limit the inner flat region was $\varepsilon=0.1$, meaning that its range is of $100 \mathrm{Mpc}$, or $z \approx 0.03$. One can clearly see above that the decay of $\langle\rho\rangle$ against an increasing $z$ is approximately linear (open circles), as expected for a scale invariant system. The straight line fitted to the points has power -0.5 , which means that the decay of the average density has fractal dimension $D=2.5$. So, despite the simplicity of the model the fractal dimension has a value not too different from what is observed. The figure also presents a plot for $d_{\ell}$ vs. $z$ (dots), showing that the linearity of the Hubble law, i.e., the distance-redshift relation, is well retained within the integration range. This proves that the apparent fractal conjecture allows for a fractal like power-law decay of the average density to co-exist with the Hubble law, this being no "paradox". 
to astronomical observations. We have started with the perturbative method proposed by Abdalla and Mohayaee (1999), where the scale factor is expanded in power series to yield perturbative terms, and then derived observational relations necessary for checking the validity of this conjecture in a perturbed spacetime, which is almost homogeneous at large values of the radial coordinate. The observational quantities derived, namely the redshift, area distance, luminosity distance, number counting, observed volume and average density, are all dependent on the solution of three ordinary first-order differential equations, which cannot be integrated analytically. Consequently, we have produced a numerical scheme for integrating these equations, namely the past radial null geodesic, the I-term, necessary for redshift evaluations, and the integrated source number count. We have found numerical solutions which show that the observed dust distribution in this perturbed Einstein-de Sitter cosmological model can be approximately described by a decaying power-law like density profile, that is, by a scale invariant, smoothed-out and averaged galaxy distribution, which is characterized by an unique and non-integer dimension. In other words, by a single fractal system. This result proves that the apparent fractal conjecture is correct, at least under the specific perturbative approach adopted here.

As consequences of our results, it is important to point out that it remains to be seen whether or not the conjecture is also valid in different, or more general, small scales perturbations to standard cosmologies. However, even if we only consider our simple perturbative approach to the problem, as described above, it is clear that the "fractal debate" currently underway (see §1) does not necessarily need to continue developing in antagonistic viewpoints. Our results suggest that the observed scale invariant pattern may be understood from within the theoretical context of standard Friedmann cosmology, and the apparent fractal conjecture may provide a starting point for developing the conceptual framework aimed at including scaling ideas, and scaling related data, into main stream cosmological research. In such a case the cosmological principle and the possibility of an infinity scale invariant, or fractal, system could, perhaps, be reconciled into a single theoretical framework. In addition, if the observed power-law density profile does appear with perturbative solutions of Friedmann cosmologies, then we may speculate that the observed scale invariant distribution of galaxies may be considered as direct observational evidence of primordial density fluctuations in the Universe, perhaps in a similar way as anisotropies of the cosmic microwave background radiation.

\section{Acknowledgements}

We would like to thank L. Pietronero for reading the original manuscript and for helpful comments and remarks. We are also grateful to L. Amendola for discussions and for pointing out a mistyping. EA wishes to thank CNPq for partial support, and FAPESP for the thematic project 97/06499-2. MBR is grateful to FUJB-UFRJ for partial support.

\footnotetext{
5 A similar conclusion has recently been reached by Joyce et al. (2000).
} 


\section{References}

Abdalla, E., Afshordi, N., Khodjasteh, K., and Mohayaee, R. 1999, Astron. Astrophys., 345,22

Abdalla, E., and Mohayaee, R. 1999, Phys. Rev. D, 59, 084014, astro-ph/9810146

Bonnor, W. B. 1974, Monthly Not. Royal Astron. Soc., 167, 55

Coleman, P. H., and Pietronero, L. 1992, Phys. Rep., 213, 311

Coles, P. 1998, Nature, 391, 120

Davis, M. et al. 1988, Astrophys. J., 333, L9

de Vaucouleurs, G. 1970, Science, 167, 1203

Ellis, G. F. R. 1971, General Relativity and Cosmology, Proc. of the International School of Physics "Enrico Fermi", R. K. Sachs, New York: Academic Press, 1971, 104

Etherington, I. M. H. 1933, Phil. Mag., 15, 761; reprinted in Gen. Rel. Grav., in press (2001)

Geller, M. 1989, Astronomy, Cosmology and Fundamental Physics, M. Caffo et al., Dordrecht: Kluwer, 1989, 83

Humphreys, N. P., Matravers, D. R., and Marteens, R. 1998, Class. Quantum Grav., 15, 3041 , gr-qc/9804025

Joyce, M., Anderson, P. W., Montuori, M., Pietronero, L., and Sylos-Labini, F. 2000, Europhys. Lett. 50, 416, astro-ph/0002504

Kihara, T., Saki, K. 1970, Publ. Astron. Soc. Japan, 22, 1

Kristian, J., and Sachs, R. K. 1966, Astrophys. J., 143, 379

Longair, M. S. 1995, The Deep Universe, Saas-Fee Advanced Course 23, B. Binggeli and R. Buser, Berlin: Springer, 1995, 317

Martínez, V. J. 1999, Science, 284, 445

Mandelbrot, B. B. 1983, The Fractal Geometry of Nature, New York: Freeman

Matravers, D. R. 1998, Proc. of the Intl. Seminar on Mathematical Cosmology, M. Rainer and H-J. Schmidt, Singapore: World Scientific, gr-qc/9808015

Peebles, P. J. E. 1980, The Large-Scale Structure of the Universe, Princeton University Press 
Pietronero, L. 1987, Physica A, 144, 257

Pietronero, L., Montuori, M., and Sylos-Labini, F. 1997, Critical Dialogues in Cosmology, N. Turok, Singapore: World Scientific, 1997, 24

Pietronero, L., and Sylos-Labini, F. 2000, astro-ph/0002124

Ribeiro, M. B. 1992a, Astrophys. J., 388, 1

Ribeiro, M. B. 1992b, Astrophys. J., 395, 29

Ribeiro, M. B. 1993, Astrophys. J., 415, 469

Ribeiro, M. B. 1994, Deterministic Chaos in General Relativity, D. Hobbil, A. Burd, and A. Coley, New York: Plenum Press, 1994, 269

Ribeiro, M. B. 1995, Astrophys. J., 441, 477, astro-ph/9910145

Ribeiro, M. B. 2001a, Fractals, in press, gr-qc/9909093

Ribeiro, M. B. 2001b, Gen. Rel. Grav., in press, astro-ph/0104181

Ribeiro, M. B., and Miguelote, A. Y. 1998, Brazilian J. Phys., 28, 132, astro-ph/9803218

Schneider, P., Ehlers, J., and Falco, E. E. 1992, Gravitational Lenses, Berlin: Springer

Sylos-Labini, F., Montuori, M., and Pietronero, L. 1998, Phys. Rep., 293, 61, astro$\mathrm{ph} / 9711073$

Turok, N. (editor) 1997, Critical Dialogues in Cosmology, Singapore: World Scientific, 1997

Weinberg, S. 1972, Gravitation and Cosmology, New York: Wiley

Wertz, J. R. 1970, Newtonian Hierarchical Cosmology, PhD thesis (University of Texas at Austin, 1970)

Wertz, J. R. 1971, Astrophys. J., 164, 227

Wu, K. K. S., Lahav, O., and Rees, M. J. 1999, Nature, 397, 225, astro-ph/9804062 